\title{
An Automatic Ship Detection Method Based on Local Gray-Level Gathering Characteristics in SAR Imagery
}

\author{
Wang XiaoLong ${ }^{1}$ and Chen CuiXia ${ }^{2}$ \\ ${ }^{1}$ Institute of Electronics, Chinese Academy of Sciences, 19, North 4th Ring Road West, Haidian District, Beijing \\ 100190, China \\ ${ }^{2}$ Institute of Biophysics, Chinese Academy of Sciences, 15, Datun Road, Chaoyang District, Beijing, 100101, China
}

Received 20th Nov 2012; accepted 28th Apr 2013

\begin{abstract}
This paper proposes an automatic ship detection method based on gray-level gathering characteristics of synthetic aperture radar (SAR) imagery. The method does not require any prior knowledge about ships and background observation. It uses a novel local gray-level gathering degree (LGGD) to characterize the spatial intensity distribution of SAR image, and then an adaptive-like LGGD thresholding and filtering scheme to detect ship targets. Experiments on real SAR images with varying sea clutter backgrounds and multiple targets situation have been conducted. The performance analysis confirms that the proposed method works well in various circumstances with high detection rate, fast detection speed and perfect shape preservation.
\end{abstract}

Key Words: Local Gray-level Gathering Degree (LGGD), Ship Detection, Synthetic Aperture Radar (SAR), Automatic Detection.

\section{Introduction}

With the increasing volume of image data that are collected from air- and space-borne SAR systems, it is becoming increasingly desirable to develop the automatic ocean targets detection technique for the expanding requirements of intelligence, surveillance and reconnaissance [1-3]. A task which is of particular importance for this is to detect ship targets in background clutters. Owing to the corner reflection from ship structures, ships are usually bright in SAR images. When ocean surface is relatively quiet, the reflection of ocean surface to radar wave is specular reflection and the echo signal is very faint. It is easy to detect these ship targets. Whereas, when the wind is fierce, large waves will be stirred. Here, the backscattering echo of ocean background will be very strong and the whole SAR image will be rather bright. Ships will be mixed with speckle noise and clutter noise, so it becomes very difficult to detect ships, especially, small ones

Many methods have been developed for detecting ships in SAR images during the last two decades, including direct ones and indirect ones [4-9]. The distributed Constant False Alarm Rate (CFAR) framework is the most widely accepted conceptual model in the direct methods [10-14]. CFAR-like detectors, as the widely used ship detection approach on which several current operational systems rely [15], involve the parameter estimations of ships and local clutters and the setting of a threshold for decision so that a constant false alarm probability is guaranteed for all values of unknown clutter parameters. The strong dependence of

Correspondence to: <xiaolong-wang@163.com>

Recommended for acceptance by $<$ Joan Serra $>$

ELCVIA ISSN: $1577-5097$

Published by Computer Vision Center / Universitat Autonoma de Barcelona, Barcelona, Spain 
CFAR schemes on prior knowledge about ships and background observation limits their application in automatic detection. Besides, due to differences in environmental conditions such as changes in clutter edge, multiple targets or jamming, the target detection is often corrupted. Another alternative technique, which firstly detects ship wakes and then seeks ships around wakes, is the indirect methods [7-9]. Generally, if ship wakes are long and evident, the ships are easier to be detected with the wakes detection methods. However, if ship wakes are not evident or absent, such as the anchorage situations, the ships can not be detected. Currently, some improved models and methods are still under development [16-19], and the related basic researches and practical problems, for automatic detection goal, still need further investigation.

Aiming to develop a simple and effective ship detection method, a "Local Gray-Level Gathering Degree (LGGD)" algorithm, motivated by the spatial distribution characteristics of cells as observed in any SAR images, is developed and then an automatic ship detector is proposed. The new detector constituted by the LGGD algorithm and a postpositive filtering scheme, is named LGGD detector. The merit of a postpositive filtering scheme rather than a prepositive one (which is generally adopted by most detectors) is: the latter always reduces target information during noise suppressing. The performance of the proposed detector has been tested on various backgrounds SAR images. The results demonstrate that the LGGD detector works well not only in the different background circumstances with high detection rate and perfect shape preservation but also in the presence of multiple scale ships and interfering targets with robust performance.

\section{Descriptions of algorithm and detector}

\subsection{LGGD algorithm}

The intensity of each resolution cell in SAR imagery denotes the radar scattering characteristics of a random ground object. Cells with different intensities and intensity distributions represent the various ground objects. Suppose that a cell $C_{1}(i, j)$ with intensity $I$ has the same observed intensity with another cell $C_{2}(i+\Delta i$, $j+\Delta j$ ), they are coherent and therefore the ground objects represented by the two cells should be the correlative objects. Accordingly, a gathering relationship of the cell pair $\left(C_{1}, C_{2}\right)$ at position $(i, j)$ can be expressed by

$$
G=1 /\left[(\Delta i)^{2}+(\Delta j)^{2}\right],\left((\Delta i)^{2}+(\Delta j)^{2} \neq 0\right)
$$

Where, $\Delta i$ and $\Delta j$ represent the spatial intervals of the cells in two directions (range and azimuth), respectively. For all cell pairs in relation to the cell $C_{1}(i, j)$, a gray-level gathering relationship of the intensity $I$ at $(i, j)$, namely gray-level gathering degree, can be computed as

$$
G_{I}(i, j)=\sum_{\Delta i=1}^{M} \sum_{\Delta j=1}^{N} 1 /\left[(\Delta i)^{2}+(\Delta j)^{2}\right],(C(i+\Delta i, j+\Delta j)=I)
$$

where $M$ and $N$ represent the spatial dimensions of the SAR image known as azimuth and range, respectively.

The $G_{I}$ describes the spatial distributions of cells and intensities, and hence it is able to highlight the changes of the spatial information. Unfortunately, its computational cost is increased with the image dimensions. To decrease computational cost, a local gray-level gathering degree is adopted by limiting a calculation range. It is obvious that the LGGD algorithm is related not only with the number of matched cells, but also with their spatial distributions. If the cells with gray-level $I$ come from the same surface of a ship target, they are gathering and hence $G_{I}$ is large, while if they come from the ocean clutter noise, they are discrete and then $G_{I}$ is small. Thus, ships should be detected if an appropriate threshold $G_{t h}$ is chosen.

\subsection{LGGD detector}

As described above, the LGGD algorithm does not require any knowledge about the operational ship and background observation after an initial calculation range (window) setting. The algorithm is sensitive to the intensity difference and is also fast enough in calculation, which is vital to build an efficient SAR detector. With these advantages, a new ship detector has been developed.

The block diagram of the proposed detector is shown in Fig.1. A square sliding window of $(2 N+1) \times$ $(2 N+1)$ pixels centred at the test cell is designed. Due to lack of ability for distinguishing bright cells from 
dark cells, the LGGD algorithm can not distinguish ships from false alarms corresponding to speckle noises, sea clutters and even dark areas (such as low-wind areas, oil spills or rain cells). As a countermeasure, a complementary filtering scheme is designed to reject false alarms. The whole detection process can be divided into three steps. Firstly, a sliding detection is executed by computing the LGGD value of each cell with its surrounding cells. When the window scans the entire image, LGGD calculation is achieved and a LGGD map is obtained. Owing to the higher intensities of ship cells, there are often no matched cells in the sliding window, that is, a zero value of $G_{I}$ is universal for ship cells. Therefore, potential target cells corresponding to the zero value of $G_{I}$ are presented at first. To reserve these cells for further discrimination, a zero-value substitution for improving their values to pass the subsequent LGGD thresholding is assigned. For example, the zero-value substitution is executed on the LDDG map by setting all the zero values to 6 (Since the value of 6 is set higher than the subsequent threshold, after the LGGD thresholding, those potential target cells can be reserved for the further discrimination). Secondly, the LGGD map is passed through a thresholding step and a binary map containing the potential targets is presented. Since any fixed threshold can not be satisfied for various situations, an adaptive-like LGGD thresholding is essential. Finally, a postpositive filtering processing is performed and false alarms in the thresholded binary map are eliminated.

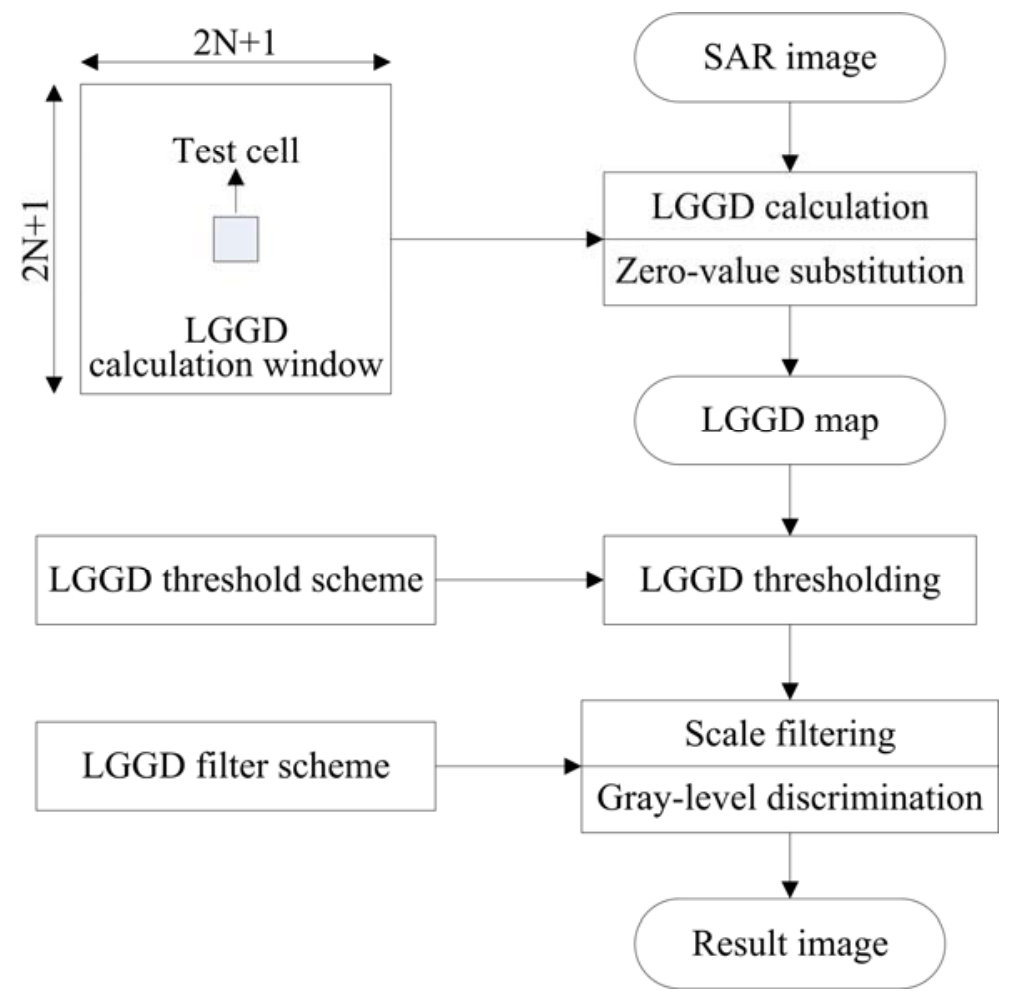

Fig.1 Block diagram of the LGGD ship detection method.

\subsubsection{Sliding window size}

As can be seen from equation (2), the calculated LGGD map depends strongly on the window size used. Too large size is disadvantageous to calculation efficiency, while too small size is not enough to calculation stability. In order to obtain a reasonable window for automatic detection, we have investigated the relationship of LGGD distribution with the increased window size by statistical analysis method. Fig.2a) shows a statistical result derived from several SAR images under different background noises (The reason for the statistical result is: although the LGGD distributions for each image are slightly different, the distribution trends are similar to each other). As can be seen, the LGGD distribution curves are steadier and all curves are close to each other when the window size is larger than 31 pixels. In addition, a theoretical LGGD calculation result is also given in Fig.2b). A series of theoretical maximum LGGD values for the increased window sizes are calculated on the assumption that their internal cells are all coherent, and then the curve is obtained by fitting these values. The curve also tends to be smooth when the window size is over 30 pixels. Thus, the size range of 30 40 pixels is suggested in our scheme. 


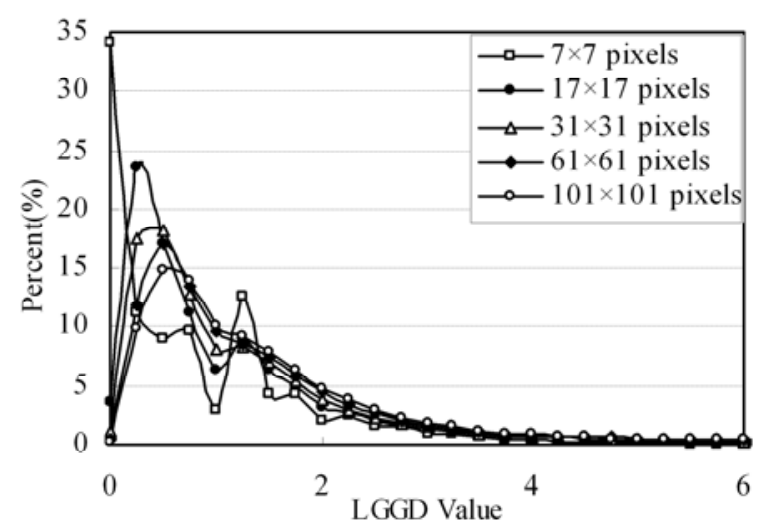

(a)

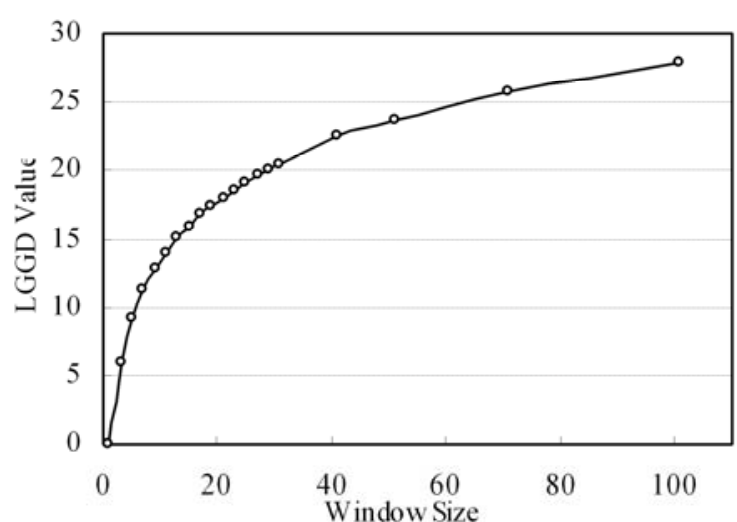

(b)

Fig.2 Dependence of LGGD on sliding window size. (a) LGGD distributions of SAR image; (b)

LGGD curve of the maximum value in sliding window.

\subsubsection{LGGD thresholding rule}

A threshold level could be fixed to discriminate ships from their background after LGGD calculation. Theoretically, due to the different LGGD distribution scopes between ships and the clutter noise, ships should be detected if an appropriate threshold $G_{t h}$ is assigned. Practically, since a threshold assigned suitable in one case can not be considered for all cases, we need to find an adaptive-like scheme of this threshold for the sake of automatic detection, which is suitable in most SAR images.

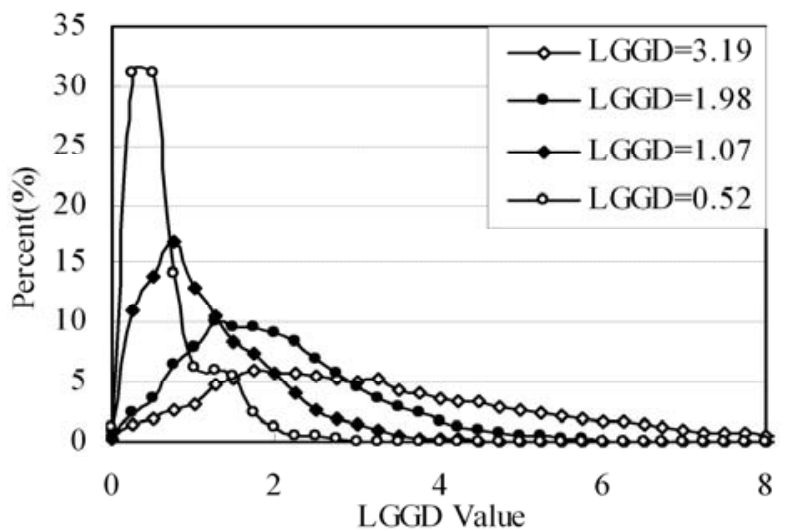

Fig.3 Relationship of the LGGD distributions with the background roughness (different LGGD values represent different background clutters).

Fig.3 shows the investigated LGGD distributions of different SAR images under different background clutters (reflected by different average LGGDs). One can see that: despite the broadened and right-shift distribution trend with the increased average LGGD, all curves tend to be smooth and near the horizontal axis around $\mathrm{LGGD}=4$, which means it is an appropriate range for threshold selection to distinguish targets from false alarms. In virtue of experimental analysis, an adaptive-like threshold rule based on the clutter level (reflected by the average LGGD value $G$ ) of SAR image is assigned for ships:

$$
G_{\text {ships }}>G_{t h} \text { with } G_{t h}=\left\{\begin{array}{cc}
3, & G<1.0 \\
4, & 1.0 \leq G \leq 2.0 \\
5, & G>2.0
\end{array}\right.
$$

Considering the heterogeneous characteristics in some large images, a sub-region processing by dividing the image into several parts is necessary for them. Then, direct to each sub-region, different thresholds based on equation (3) should be adopted since each sub-region has different $G$ value.

\subsubsection{LGGD filtering scheme}


Despite its sensitivity to intensity difference, the LGGD algorithm can not distinguish between bright cells and dark cells. As reflected in the thresholded binary map (Fig.4b)), there are still lots of discrete dots (corresponding to speckle noises and sea clutters) and spots (corresponding to some dark areas, e.g., lowwind areas, oil spills or rain cells) [20], which can not be eliminated by the LGGD thresholding.

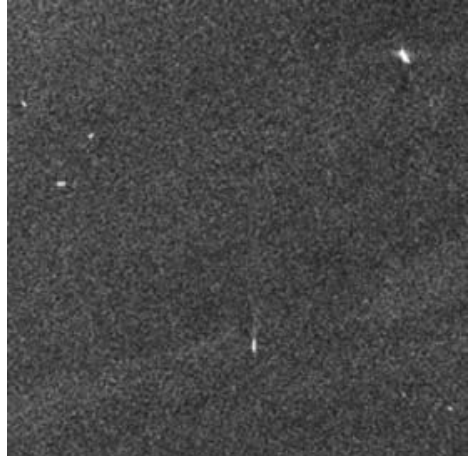

(a)

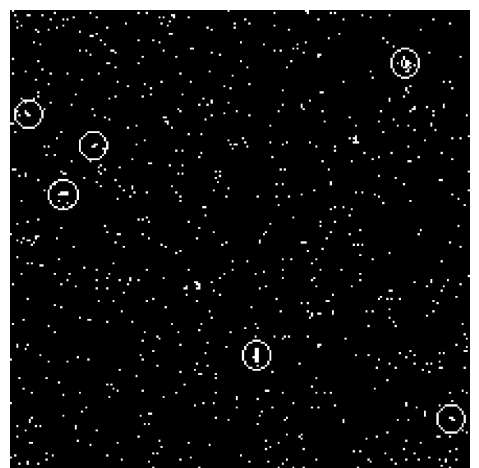

(b)

Fig.4 Characteristics of the LGGD thresholded binary map. (a) Original SAR image; (b) LGGD thresholded binary map.

In order to remove them, we have adopted a simple and effective complementary filter, which is constituted by a scale-based filter (for discrete dots) and a cell-based gray-level discrimination rule (for dark cells). The filter adopts two alternative windows, $3 \times 3$ pixels and $5 \times 5$ pixels, to handle two types of binary maps derived from weak and strong noise background SAR images respectively. For the former, if the number of adjacent bright cells in the window is greater than or equal to 3, they will be retained for further discrimination; for the latter, the number is 5. Complementarily, an empirical gray-level discrimination threshold $I_{t h}$ based on the average intensity $I_{a}$ of the inputted image to identify ship cells:

$$
I_{\text {th }}>\left\{\begin{array}{cc}
100, & I_{a}<50 \\
50+I_{a}, & 50 \leq I_{a} \leq 100 \\
I_{a}+\left(200-I_{a}\right) / 2, & 100 \leq I_{a} \leq 200 \\
I_{a} & I_{a} \geq 200
\end{array}\right.
$$

\section{Experimental results and analysis}

The proposed detector has been applied on a series of real SAR images, considering three crucial situations, for evaluating its performances: 1) strong noise background situation; 2) heterogeneous background situation; and 3) multiple targets situation (including multiple-scale targets situation and closely separated targets situation). Since the corresponding ground truth data were not available, a precise crosscheck could not be performed. The validation of the detection results was based on the visual inspection. For comparison, the two-parameter CFAR method[14], as the widely used ship detection algorithm on which several current operational systems rely, is used, and the corresponding results at a false alarm rate of $0.05 \%$ are presented.

\subsection{Strong noise background situation}

Under weak noise background, most detectors, such as CFAR-like detectors, can achieve high detection rate and low false alarm rate. However, things are different in strong noise cases. Ships will be mixed with the ocean clutters due to strong backscattering echo of them, which bring many difficulties for the detection of them.

To test this situation, an ALOS HH polarized L-band SAR image $(280 \times 400$ pixels, $10 \mathrm{~m}$ pixel size $)$ quoted from the literature [21], as shown in Fig. 5a), is adopted in this paper. To compare the results, we also quoted its CFAR detection result $(0.05 \%$ false alarm rate, Fig. $5 b))$. Fig. $5 c)$ shows the LGGD detection result. As can be seen, the hidden ships can be detected perfectly by our method without producing any false 
alarm. Whereas, the conventional CFAR method would cause lots of false alarms even though it works at a very low false alarm rate.

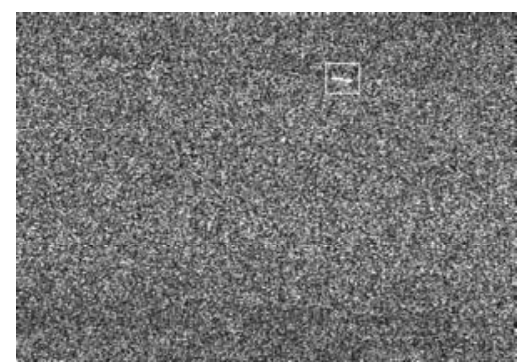

(a)

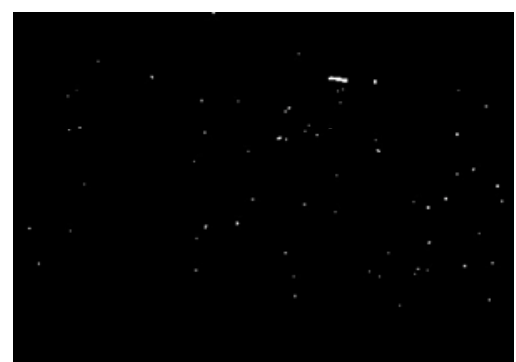

(b)

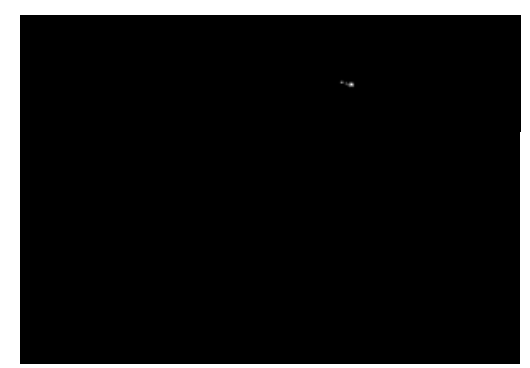

(c)

Fig.5 Detection results for strong speckle situation. (a) Original SAR image; (b) Two-parameter CFAR result; (c) LGGD result $(31 \times 31$ pixels window).

\subsection{Heterogeneous background situation}

Performance under heterogeneous background condition is a very important evaluation criterion to any target detector. Generally, ships are brighter than background clutters in marine SAR images, since the scattering of ship targets can last longer than sea clutters in azimuth. This is helpful to detect ship targets. But when the size of a ship is small or the scattering of a ship is faint, it is rather difficult to separate the ship target from the non-homogeneous background clutters.

Fig. 6a) gives a typical ERS C-band SAR image $(500 \times 500$ pixels, $12.5 \mathrm{~m}$ pixel size $)$ near the coast of Singapore containing 5 ships in heterogeneous regions, and Fig. 6b) and c) present the detection results by the CFAR method and the LGGD method respectively. Although there are some sea clutters within the heavily heterogeneous regions mistaken to be ships, the detection result by our LGGD method, compared with that of the CFAR method, is acceptable. In fact, a perfect LGGD detection result, as shown in Fig. 6d), can be achieved without regard to the auto-detection goal when the sliding window is widened from 31 pixels to 101 pixels. However, as has been mentioned above, the larger window will lower the calculation efficiency. The computational cost is $0.688 \mathrm{~s}$ for 31 pixels window (and $1.828 \mathrm{~s}$ for CFAR method), while it reaches 5.352s when the window size is widened to 101 pixels. The experiment validates the detection ability of the LGGD method for the heterogeneous background situations.

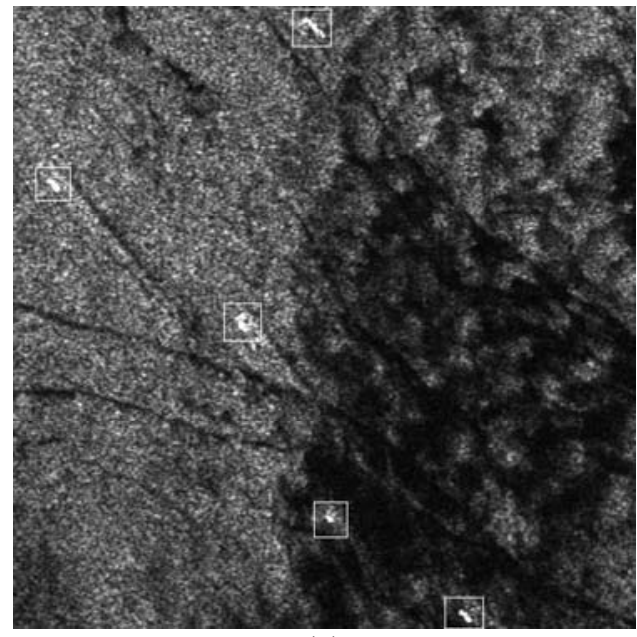

(a)

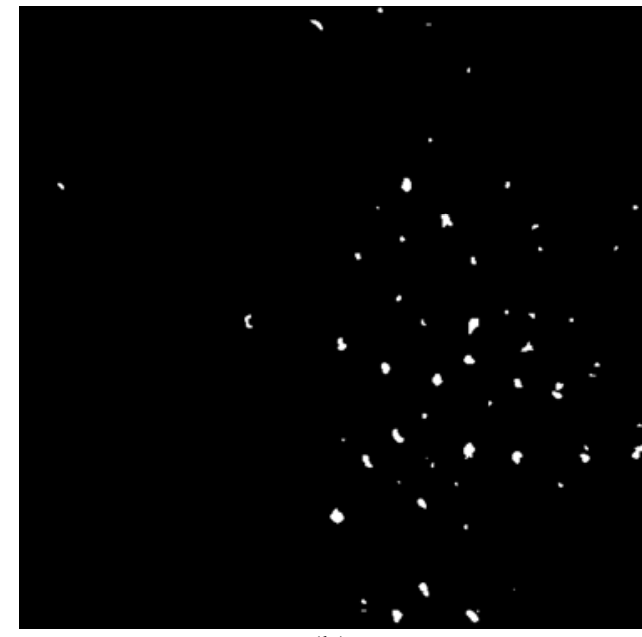

(b) 


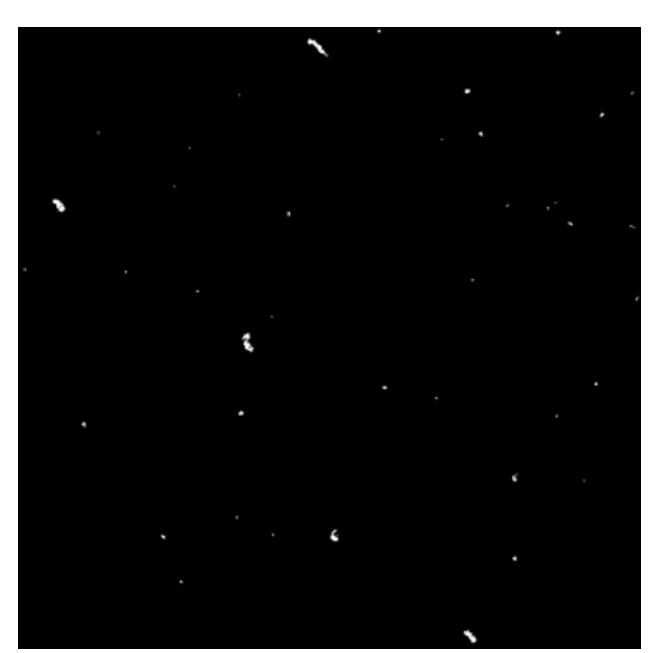

(c)

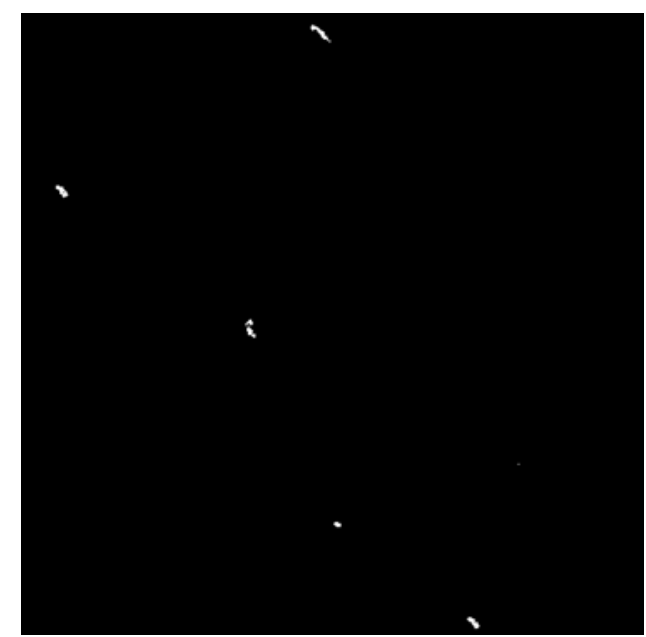

(d)

Fig.6 Detection results for non-homogeneous background situation. (a) Original SAR image; (b) CFAR result; (c) LGGD result ( $31 \times 31$ pixels window); (d) LGGD result $(101 \times 101$ pixels window).

\subsection{Multiple targets situation}

The multiple targets tasks, including situations for multiple-scale targets and closely separated targets, are always a challenge for most detectors, especially for CFAR-like detectors, since their performances are strong dependent on the sizes of the detection windows [22]. The various ship scales often make it difficult to determine an appropriate window to satisfy all expected target sizes in such situation. The case can be seen in Fig. 7. Fig. 7a) shows a C-band ERS SAR image of a busy port $(400 \times 344$ pixels, $12.5 \mathrm{~m}$ pixel size $)$ within heterogeneous regions in which 50 ships are present. It should be a difficult task since different scales of ships are included in the image, including some small ships, faint scattering ships and hidden ships. Fig. $7 \mathrm{~b}$ ) and c) presents the detection results by the CFAR detector and the LGGD detector, respectively. As can be seen, excepting for the only one false alarm as encircled by white circle in Fig. 7c), the LGGD detector highlights all ships and detects them perfectly either in shapes or in sizes, which is vital to the further estimations of the ship parameters, including length, width and orientation [23]. In contrast, the CFAR detector not only causes many false alarms but also misses two faint ships labeled as 1 and 2 in Fig. 7a). Furthermore, the bad shape preservation, as the worst detection representative of ship with the signature of 3 in Fig. 7a), also reveals its insufficiency to such situations in comparison with our method.

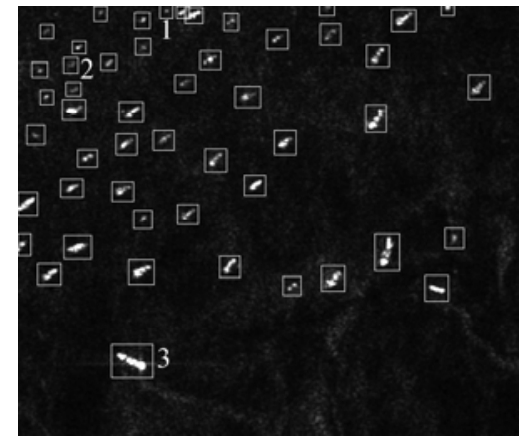

(a)

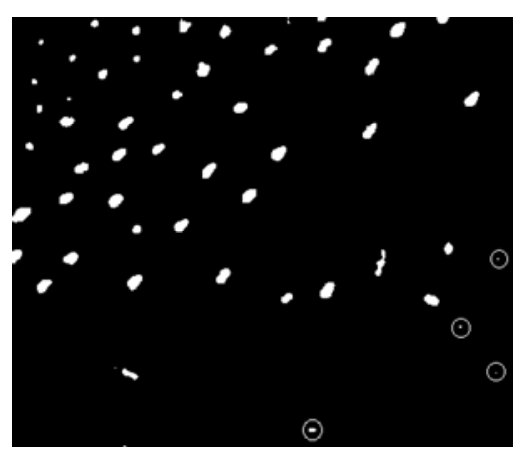

(b)

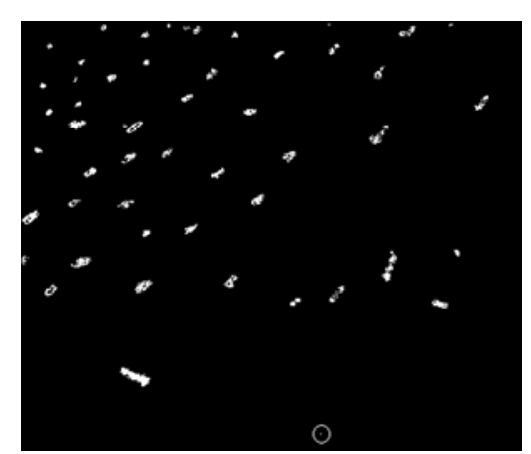

(c)

Fig.7 Detection results for multiple scale targets situation. (a) Original SAR image; (b) CFAR result; (c) LGGD result $(31 \times 31$ pixels window).

Some closely separated targets or jamming lead to another detection problem for CFAR-like detectors due to one or more interfering targets occupy some of the reference window cells [10], which results in a recurring detection loss in ship gathering regions. To illustrate the situation, an ERS-2 SAR image of Suez Bay in north of the Red Sea (Fig. 8a), $240 \times 244$ pixels, $12.5 \mathrm{~m}$ pixel size) within white box in which 5 ships jammed is adopted. The corresponding detection results by the CFAR method and the LGGD method are shown in Fig. 8b) and c). As can be seen, the CFAR method misses one ship in the jamming area, while the 
LGGD method finds all five ships successfully, which means that the LGGD method has an adequate level of immunity to interference.

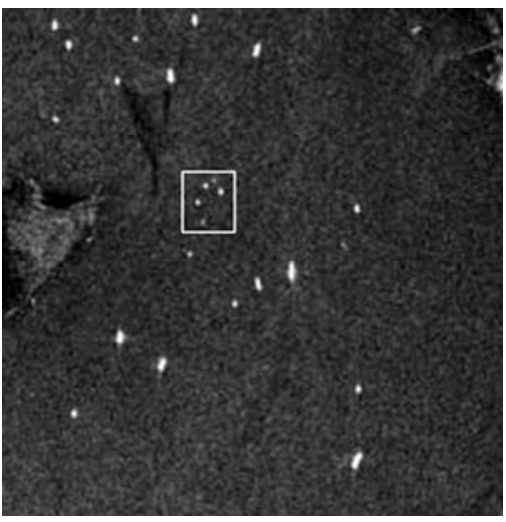

(a)

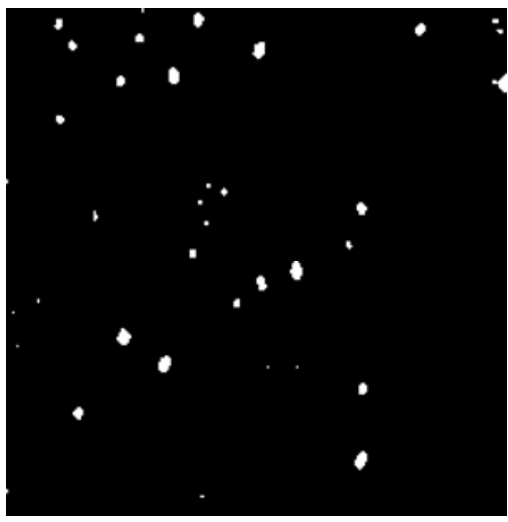

(b)

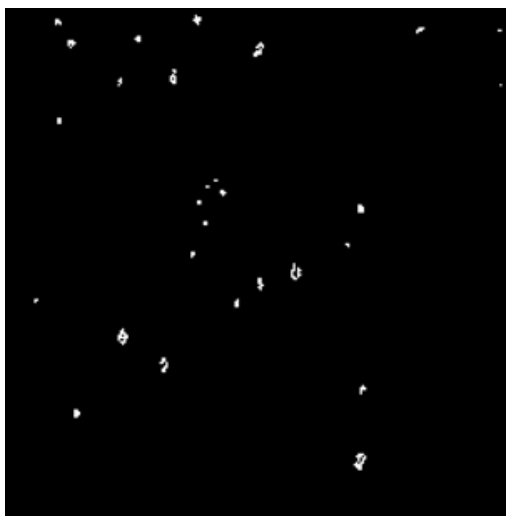

(c)

Fig.8 Detection results for closely separated targets situation. (a) Original SAR image; (b) CFAR result;

(c) LGGD result $(31 \times 31$ pixels window).

\section{Conclusions}

A LGGD algorithm for ship detection from SAR imagery is proposed in this paper, the algorithm uses the local gray-level gathering characteristics of SAR imagery to characterize its spatial intensity distribution, independent of any prior knowledge about ships and background observation, hence is able to execute automatic detection. Based on this, a simple and effective detector for ship targets, LGGD for short, has been developed. The method provides a great benefit to the faint target detection under the higher sea state conditions.

Compared with the CFAR-like methods, the merits of the LGGD method can be concluded as: 1) the method does not require any prior knowledge therefore is able to perform automatic detection; 2) the method has an adequate level of immunity to interference, which is more suitable for applying in the multiple targets detection; and 3) the method has strong differentiation ability for the target edge due to its sensitiveness to the intensity difference hence can achieve perfect shape preservation. In fact, the LGGD method, in virtue of some primary tests, is nearly always effective to the gray images due to its gray-level dependence, including Panchromatic (PAN) images of optical images. However, the validity of the LGGD method still need to be further verified in terrestrial SAR images and optical images for its wider applicability, although it was developed for addressing the clutter interferences in ship detection and only marine SAR images with low background complexity were tested.

\section{Acknowledgments}

This work was supported by grants from the National High Technology Research and Development Program of China (No. 2008AA121805-1) and the National Science Foundation of China (No. 61072113 and No. 61101201)

\section{References}

[1] M. Yeremy, J. W. M.Campbell, K. Mattar, T. Potter, "Ocean surveillance with polarimetric SAR", Can. J. Remote Sens., 27(4):328-344, 2001.

[2] T. Mariv, L. Carlos, J.M. Jordi, "Automatic vessel monitoring with single and multidimensional SAR images in the wavelet domain", J. Photo. Remote Sens., 61(3-4):260-278, 2006.

[3] Y. Liu, M. Xing, R. Guo, L. Zhang, X. Bai, Z. Bao, "Sandglass transformation for synthetic aperture radar detection and imaging of ship at low signal-to-clutter-plus-noise ratio", IET Radar Sonar Navig., 5(3):361-373, 2011. 
[4] M. Tello, et al, "Automatic vessel monitoring with single and multidimensional SAR images in the wavelet domain”, Isprs J. Photo. Remote Sens., 61(3):260-278, 2006.

[5] M. S. Greco, F. Gini, "Statistical analysis of high-resolution SAR ground clutter data", IEEE Trans. GRS, 45(3):566-575, 2007.

[6] M. Liao, C. Wang, Y. Wang, L. Jiang, "Using SAR images to detect ships from sea clutter", IEEE Trans. GRSL, 5(2):194-198, 2008.

[7] J. Chen, J. Y. Sun, B. Chen, S. Q. Xu, "Ship wake detection based on nonsubsampled contourlet", Infrared Laser Eng., 6(4):972-976, 2007.

[8] M. V. Dragosevic, P. W. Vachon, "Estimation of ship radial speed by adaptive processing of RADARSAT-1 fine mode data", IEEE Trans. GRSL, 5(4):678-682, 2008.

[9] P. Wu, J. Wang, Y. X. Zhang, H. X. Duan, "An improved method of ship wake detection based on localized radon transform", IET Int. Radar Conf., Guilin, China, 1-4, 2009.

[10] H. Zoheir, S. Faouzi, "Distributed IVI-CFAR detection in non-homogeneous environments", Signal Process., 84(7):1231-1237, 2007.

[11] Z. Amir, N. Yaser, "Automatic dual censoring cell-averaging CFAR detector in non-homogenous environments", Signal Process., 88(11):2611-2621, 2008.

[12] G. Gao, L. Liu, L. J. Zhao, G. T. Shi, G.Y. Kuang, "An adaptive and fast CFAR algorithm based on automatic censoring for target detection in high-resolution SAR images", IEEE Trans. GRS, 47(6):1685-1696, 2009.

[13] X. W. Meng, "Performance analysis of ordered-statistic greatest of constant false alarm rate with binary integration for M-sweeps", IET Radar Sonar Navig., 4(1):37-48, 2010.

[14] Y. G. Ji, J. Zhang, J. M. Meng, X. Zhang, "A new CFAR ship target detection method in SAR imagery”, Acta Oceanol. Sin., 29(1):12-16, 2010.

[15] T. Liu, G. Lampropoulos, "A new polarimetric CFAR ship detection system", IEEE IGARSS 2006, Denver, USA, 137-140, 2006.

[16] F. Zhang, B. Wu, "A scheme for ship detection in inhomogeneous regions based on segmentation of SAR images”, Int. J. Remote Sens., 29(19):5733-5747, 2008.

[17] A. Gambardella, F. Nunziata, M. Migliaccio, "A physical full-resolution SAR ship detection filter", IEEE Trans. GRSL, 5(4):760-763, 2008.

[18] S. Q. Huang, D. Z. Liu, G. Q. Gao, X. J. Guo, “A novel method for speckle noise reduction and ship target detection in SAR images", Pattern Recog., 2(7):1533-1542, 2009.

[19] J. Chen, Y. L. Chen, J. Yang, "Ship detection using polarization cross-entropy", IEEE Trans. GRSL, 6(4):723-727, 2009.

[20] Y. M. Shu, J. Li, H. Yousif, G. Gomes, "Dark-spot detection from SAR intensity imagery with spatial density thresholding for oil-spill monitoring", Remote Sens. Envir., 114:2026-2035, 2010.

[21] Y. Yu, Z. H. Ding, B. Wang, L. M. Zhang, "Visual attention-based ship detection in SAR images", Adv. Neur. Net. Res. Appli., 67(4):283-292, 2010.

[22] C. H. Jung, H. J. Yang, Y. K. Kwag, "Local cell-averaging fast CFAR for multi-target detection in high-resolution SAR images", 2nd Asian-Pacific Conf. SAR (APSAR), Xian, China, 206-209, 2009.

[23] P. W. Vachon, R. A. English, J. Wolfe, "Ship signatures in synthetic aperture radar imagery", IEEE IGARSS 2007, Barcelona, Spain, 1393-1396, 2007. 